\title{
Approximation operator based on neighborhood systems
}

\author{
Pei Wang ${ }^{\mathrm{a}}$, Qingjun $\mathrm{Wu}^{\mathrm{b}, *}$, Jiali He $\mathrm{He}^{\mathrm{b}, *}$, Xiao Shang ${ }^{\mathrm{c}}$ \\ ${ }^{a}$ Guangxi Universities Key Lab of Complex System Optimization and Big Data Processing, Yulin Normal \\ University, Yulin 537000, China \\ ${ }^{b}$ School of Mathematics and Information Science, Yulin Normal University, Yulin 537000, China \\ c Yulin Normal University, Yulin 537000, China
}

\begin{abstract}
In this paper, we propose a new covering-based set in which the lower and the upper approximation operations are defined by neighborhood systems. We discuss this new type of covering-based set systematically in two steps. First, we study the basic properties of this covering-based set, such as the properties of normality, contraction, and monotone. Second, we discuss the relationship between the new type of covering-based set and the other ten sets proposed.
\end{abstract}

Keywords: rough sets, Covering approximation space, Neighborhood system, approximation operation, Partition.

\section{Introduction}

Pawlak proposed the concept of rough set and did some works [11]. It is a useful and powerful tool for handing uncertain things. It has been widely used in process control, economics, medical diagnosis, biochemistry, environmental science, biology, chemistry, psychology, conflict analysis, and so on. Comparing with other methods, the rough set theory has its advantages. For example, it does not need any additional information about data in the process of uncertain data. From then on, many researchers have made many significant contributions to developing the rough theory $[11,13,15,17,18,19,20,21,22,23,24,25,26]$. However, a problem with Pawlak's rough set theory is that partition or equivalence relation is explicitly used in the definition of the lower and upper approximations. The partition or equivalence relation is hard to be satisfied since it has its limitations. It can only deal with complete information systems. To address this issue, one approach was to extend equivalence relations to tolerance relation or general relation. Another important approach was to relax the equivalence relation to a covering and got the covering-based rough sets. In 1983, Zakowski first generalized the classical rough set theory using coverings of a universe instead of partition or equivalence relation [27]. Such generalization leads to various covering approximation operators that are both theoretical and practical importance [28, 29, 30]. The relationships between properties of covering-based approximation and their corresponding coverings have attracted intensive research. How to obtain this useful information and deal with uncertain data has become a widely studied problem. In the process of solving the problem, many methods

\footnotetext{
* Corresponding author

Email addresses: wangpei131@sina.com.cn (Pei Wang), dexian2017@hnu.edu.cn (Qingjun Wu), hjljuly@163.com. (Jiali He), 443571909@qq.com (Xiao Shang)
} 
have been proposed, such as statistical methods, fuzzy set theory $[9,16]$, computing word [16], and rough set theory $[12,14]$.

In the following, Bonikowski et al. studied this type of covering-based rough sets from the viewpoint of formal concepts. At this time, covering-based rough sets as a more powerful tool, it can be used to deal with the problems which can not be solved by classical Rough set theory. Such as the granularity problems in information systems. Up to now, about ten types of covering-based rough sets have been proposed and studied $[10,19,21]$. and many scholars did a lot of works on it. T. Yang et. al. discuss the reduction of covering [31]. J. Zhan et. al. investigated two types of coverings based multigranulation rough fuzzy sets and certain types of soft coverings based rough sets $[32,33,35]$. L. Zhang et. al. obtained novel class of fuzzy soft $\beta$-coverings-based fuzzy rough sets with applications to multi-criteria fuzzy group decision making [34]. D'eer et. al. studied the neighborhood operators for covering-based rough sets [36, 37]. José et. al. considered fuzzy techniques for decision making and some formal relationships among soft sets, fuzzy sets, and their extensions [38, 39]. Przemyslaw Grzegorzewski discussed the separability of Fuzzy relations [40].

In this paper, a new type of covering-based rough set is proposed. This paper is arranged as follow: In section 2, the properties such as normality, contraction, and monotone are studied. If a property does not hold, the necessary and sufficient condition about neighborhood system in which this property holds is researched. In section 3, the condition that the type of covering-based rough set equals the other ten sets proposed by other scholars is evaluated and discussed.

\section{The definition and properties of covering-based approximation operators}

Let $U$ be a finite set, called a universe, $R$ be a equivalence relation on $U$, then the partition induced by $R$ is denoted by $U / R=\left\{X_{1}, X_{2}, \cdots X_{n}\right\}$. For any $X \subseteq U$, two subsets of $\mathrm{U}$ are given as follows:

$$
\begin{aligned}
& \underline{R}(X)=\cup\{K \in C: K \subseteq X\} \\
& \bar{R}(X)=\cup\{K \in C: K \cap X \neq \emptyset\}
\end{aligned}
$$

The first subset $\underline{R}(X)$ and the second $\bar{R}(X)$ are called lower approximation and upper approximation of $X$ respectively.

Obviously, a partition of $U$ is a covering of $U$, but a covering of $U$ is not necessarily a partition of $U$, so the definition of covering approximation space has been introduced. Before definite the new type of covering-based rough set, it is necessary for us to give some basic definitions about covering approximation space.

Definition 2.1 (Covering[25]). Let $U$ be a universe, a set of non-empty subsets $C=\left\{K_{i} \subseteq U\right.$ : $i \in I\}$ is called a covering of $U$ if it satisfies $\cup C=U$, and $K_{i} \neq \varnothing$ for each $i \in I$.

Definition 2.2 (Covering approximation space[23]). Let $U$ be a universe, $C$ a covering of $U$, then we call $U$ together with covering $C$ a covering approximation space, denoted by $(U, C)$.

Definition 2.3 (The neighborhood of a point $x[24]$ ). Let $(U, C)$ be a Covering approximation space. For $x \in U$, we call $N(x)=\cap\{K \in C: x \in K\}$ the neighborhood of point $x$.

Definition 2.4 (Neighborhood system[24]). Let $(U, C)$ be a Covering approximation space. We call $N=\{N(x): x \in U\}$ the neighborhood system induced by $(U, C)$. 
From now on, symbol $(U, C, N)$ is used to represent a Covering approximation space $(U, C), N$ is the neighborhood system induced by $(U, C)$.

Lemma 2.1. [24] Let $(U, C, N)$ be a Covering approximation space. If $x, y \in U$ such that $x \in N(y)$, then $N(x) \subseteq N(y)$.

Proposition 2.1. Let $(U, C, N)$ be a Covering approximation space. $N$ froms a partition of $U \Leftrightarrow$ there does not exist a pair $x, y \in U$ such that $x \in N(y)$ and $y \notin N(x)$.

Proof. Necessity is simple, we only need to prove the sufficiency. Suppose there does not exist a pair $x, y \in U$ such that $x \in N(y)$ and $y \notin N(x)$, but $N$ is not a partition of $U$. We take two conditions into consideration: (1) $\exists x_{0}, y_{0} \in U$ such that $x_{0} \in N\left(y_{0}\right)$ and $y_{0} \notin N\left(x_{0}\right)$. It is a contradiction to the assumption. (2) $\exists x_{1}, y_{1} \in U$ such that $N\left(x_{1}\right) \cap N\left(y_{1}\right) \neq \emptyset, x_{1} \notin N\left(y_{1}\right)$ and $y_{1} \notin N\left(x_{1}\right)$. Select $z_{1} \in N\left(x_{1}\right) \cap N\left(y_{1}\right)$, since $y_{1} \notin N\left(x_{1}\right)$, by lemma 2.1 , we obtain a pair $y_{1}, z_{1} \in U$ such that $z_{1} \in N\left(y_{1}\right)$ and $y_{1} \notin N\left(z_{1}\right)$. It is also a contradiction to the assumption. From condition (1)(2), the proof of the sufficiency is completed.

Definition 2.5 (Membership of a point $x$ ). [23] Let $(U, C, N)$ be a Covering approximation space. For a point $x \in U, F M(x)=\{K \in C: x \in K\}$ is called the membership of $x$.

Definition 2.6 (Minimal description of a point $x$ ). [23] Let $(U, C, N)$ be a Covering approximation space. The minimal description of a point $x$ is defined as

$$
M d(x)=\{K \in C: x \in K \in C \wedge(\forall S \in C \wedge x \in S \subseteq K \Rightarrow K=S)\}
$$

Definition 2.7. Let $(U, C, N)$ be a Covering approximation space. For $X \subseteq U$, the covering-based lower approximation operation $N: 2^{U} \rightarrow 2^{U}$ is defined as

$$
\underline{N}(X)=\cup\{N(x): N(x) \subseteq X\}
$$

And the covering-based upper approximation operation $N: 2^{U} \rightarrow 2^{U}$ is defined as

$$
\bar{N}(X)=\underline{N}(X) \cup\{x \in U: N(x) \cap(X-\underline{N}(X)) \neq \emptyset\}
$$

Definition 2.8. Let $(U, C, N)$ be a Covering approximation space. For $X \subseteq U$,

(1) If $\underline{N}(X)=X$, then $X$ is called an inner definable subset.

(2) If $\overline{\bar{N}}(X)=X$, then $X$ is called an outer definable subset.

(3) If $\bar{N}(X)=X=\underline{N}(X)$, then $X$ is called a definable subset.

Theorem 2.1 below describe what is the essence of inner definable subset, outer definable subset and definable subset.

Theorem 2.1. Let $(U, C, N)$ be a Covering approximation space. For $X \subseteq U$,

(1) $X$ is an inner definable subset $\Leftrightarrow \exists A \subseteq U$ such that $X=\cup\{N(x): x \in A\}$.

(2) $X$ an outer definable subset $\Leftrightarrow \forall x \notin X \Rightarrow(N(x) \cap X) \subseteq \underline{N}(X)$.

(3) $X$ is a definable subset $\Leftrightarrow X$ is an inner definable subset.

Proof. The proof is simple.

Remark 2.1. $\mathrm{X}$ is a definable subset $\Leftrightarrow \mathrm{X}$ is an inner definable subset $\Rightarrow \mathrm{X}$ is an outer definable subset, but $\mathrm{X}$ is an outer definable subset $\nRightarrow \mathrm{X}$ is an inner definable subset. 
Example 2.1. If let $U=\{1,2,3,4,5\}, C=\{\{1,2\},\{3,4\},\{4\},\{5\}\}, X_{0}=\{1,2,3\}$, then $\underline{N}\left(X_{0}\right)=$ $\{1,2\} \neq X_{0}$, but $\bar{N}\left(X_{0}\right)=\{1,2,3\}=X_{0}$.

Proposition 2.2. Let $(U, C, N)$ be a Covering approximation space. $\forall X, Y \subseteq U$, we have:

$$
\begin{aligned}
& (1) \underline{N}(U)=U(C o-\text { normality }) \\
& (2) \bar{N}(U)=U(C o-\text { normality }) \\
& (3) \underline{N}(\emptyset)=\emptyset(\text { Normality }) \\
& (4) \bar{N}(\emptyset)=\emptyset(\text { Normality }) \\
& (5) \underline{N}(X) \subseteq X \subseteq \bar{N}(X)(\text { Contraction - Extension }) \\
& (6) \underline{N}(X \cap Y)=\underline{N}(X) \cap \underline{N}(Y)(\text { Multiplication }) \\
& (7) X \subseteq Y \Rightarrow \underline{N}(X) \subseteq \underline{N}(Y)(\text { Monotone }) \\
& (8) \underline{N}(\underline{N}(X))=\underline{N}(X)(\text { Idempotency }) \\
& (9) \bar{N}(\bar{N}(X))=\bar{N}(X)(\text { Idempotency }) \\
& (10) \underline{N}(X) \cup \underline{N}(Y) \subseteq \underline{N}(X \cup Y) \\
& (11) \bar{N}(X \cup Y) \subseteq \bar{N}(X) \cup \bar{N}(Y) .
\end{aligned}
$$

Proof. The proofs of (1) - (7), (10)(11) are obvious. We only prove (8),(9).

Firstly, we prove (8). From proposition 2.2 property (5), $\underline{N}(\underline{N}(X)) \subseteq \underline{N}(X)$ holds. $\forall y \in \underline{N}(X)$, since $\underline{N}(X)=\cup\{N(x): N(x) \subseteq X\}$, so $N(y) \subseteq \underline{N}(X)$. By the definition of $\underline{N}(\underline{N}(X))$, we have $y \in \underline{N}(\underline{N}(X))$. This means $\underline{N}(X) \subseteq \underline{N}(\underline{N}(X))$, combining $\underline{N}(\underline{N}(X)) \subseteq \underline{N}(X)$, the proof of property (8) is completed.

Secondly, we prove property (9). From proposition 2.2 property (5), $\bar{N}(X) \subseteq \bar{N}(\bar{N}(X))$ holds. $\forall x \in \bar{N}(\bar{N}(X))$, we take two conditions into consideration: (a) $x \in \underline{N}(\bar{N}(X))$, we have $x \in$ $\bar{N}(X))$. (b) $N(x) \cap(\bar{N}(X)-\underline{N}(\bar{N}(X))) \neq \emptyset$, select $x_{0} \in N(x) \cap(\bar{N}(X)-\underline{N}(\bar{N}(X)))$. Since $x_{0} \in \bar{N}(X)-\underline{N}(\bar{N}(X))$, so $x_{0} \notin \underline{N}(X)$ and $N\left(x_{0}\right) \cap(X-\underline{N}(X)) \neq \emptyset$. On the other hand, from the condition that $x_{0} \in N(x)$ and Lemma 2.1, we have $N(x) \cap(X-\underline{N}(X)) \neq \emptyset$. This means $x \in \bar{N}(X)$. According to $(a)(b)$, the proof of $(9)$ is completed.

Generally speaking, suppose $(U, C, N)$ be a Covering approximation space. ( ()$X \subseteq Y \subseteq U \nRightarrow$ $\bar{N}(X) \subseteq \bar{N}(Y),(\sharp) \bar{N}(X \cup Y)=\bar{N}(X) \cup \bar{N}(Y)$ does not always hold.

Example 2.2. Let $U=\{1,2,3,4,5,6\}, C=\{\{1\},\{2\},\{3\},\{1,2,3,4,5\},\{1,2,3,4,5,6\}\}, X_{0}=$ $\{1,2,3,4\}, Y_{0}=\{1,2,3,4,5\}$, then $X_{0} \subseteq Y_{0}, \bar{N}\left(X_{0}\right)=\{1,2,3,4,5,6\} \nsubseteq \bar{N}\left(Y_{0}\right)=\{1,2,3,4,5\}$ and $\bar{N}\left(X_{0} \cup Y_{0}\right)=\{1,2,3,4,5\} \neq \bar{N}\left(X_{0}\right) \cup \bar{N}\left(Y_{0}\right)=\{1,2,3,4,5,6\}$.

Theorem 2.2. Let $(U, C, N)$ be a Covering approximation space. $\forall X \forall Y[(X \subseteq Y \subseteq U) \Rightarrow$ $(\bar{N}(X) \subseteq \bar{N}(Y))] \Leftrightarrow \forall X \forall Y(\bar{N}(X \cup Y)=\bar{N}(X) \cup \bar{N}(Y))$

Proof. " $\Rightarrow$ ". $\forall X, Y \subseteq U$, since $X, Y \subseteq(X \cup Y)$, so $\bar{N}(X) \cup \bar{N}(Y) \subseteq \bar{N}(X \cup Y)$. By proposition 2.2(11), we have $\bar{N}(X \cup Y)=\bar{N}(X) \cup \bar{N}(Y)$.

"Е". $\forall X \subseteq Y \subseteq U$, since $X \cup Y=Y$, so $\bar{N}(Y)=\bar{N}(X \cup Y)=\bar{N}(X) \cup \bar{N}(Y)$. This means $\bar{N}(X) \subseteq \bar{N}(Y))$.

Theorem 2.3. Let $(U, C, N)$ be a Covering approximation space. $\forall X \forall Y[(X \subseteq Y \subseteq U) \Rightarrow$ $(\bar{N}(X) \subseteq \bar{N}(Y))] \Leftrightarrow$ There does not exist a pair $x, y[(|N(x)|>1) \wedge(|N(y)|>1) \wedge(x \in N(y)) \wedge(y \notin$ $N(x))]$. 
Proof. " $\Rightarrow$ ", proof by contradiction. Suppose $\exists x_{0} \exists y_{0}\left[\left(\left|N\left(x_{0}\right)\right|>1\right) \wedge\left(\left|N\left(y_{0}\right)\right|>1\right) \wedge\left(x_{0} \in N\left(y_{0}\right)\right) \wedge\right.$ $\left.\left(y_{0} \notin N\left(x_{0}\right)\right)\right]$. Select $z_{0} \in N\left(x_{0}\right)$ and $x_{0} \neq z_{0}$, let $X_{0}=N\left(x_{0}\right)-\left\{z_{0}\right\}$ and $Y_{0}=N\left(x_{0}\right)$. We can obtain the fact that $X_{0} \subseteq Y_{0}, y_{0} \in \bar{N}\left(X_{0}\right)$ and $y_{0} \notin \bar{N}\left(Y_{0}\right)$. This means $\bar{N}\left(X_{0}\right) \nsubseteq \bar{N}\left(Y_{0}\right)$, contradicts the assumption of necessity.

$" \Leftarrow$ ", proof by contradiction. Suppose $\exists X_{0} \exists Y_{0} \exists p_{0}\left[\left(X_{0} \subseteq Y_{0}\right) \wedge\left(p_{0} \in \bar{N}\left(X_{0}\right)\right) \wedge\left(p_{0} \notin \bar{N}\left(Y_{0}\right)\right)\right]$. Since $p_{0} \notin \bar{N}\left(Y_{0}\right)$, so $p_{0} \notin Y_{0}$ and $p_{0} \notin X_{0}$. From the fact that $p_{0} \in \bar{N}\left(X_{0}\right)$, we have $N\left(p_{0}\right) \cap$ $\left(X_{0}-\underline{N}\left(X_{0}\right)\right) \neq \emptyset$. Select $q_{0} \in N\left(p_{0}\right) \cap\left(X_{0}-\underline{N}\left(X_{0}\right)\right)$, take the conditions $p_{0} \notin X_{0}$ and $p_{0} \notin$ $\bar{N}\left(Y_{0}\right)$ into consideration, we have $p_{0} \neq q_{0}, q_{0} \in N\left(p_{0}\right),\left|N\left(q_{0}\right)\right|>1$ and $p_{0} \notin N\left(q_{0}\right)$. This means $p_{0}, q_{0}\left[\left(\left|N\left(p_{0}\right)\right|>1\right) \wedge\left(\left|N\left(q_{0}\right)\right|>1\right) \wedge\left(q_{0} \in N\left(p_{0}\right)\right) \wedge\left(p_{0} \notin N\left(q_{0}\right)\right)\right]$, contradicts the assumption of sufficiency.

Corollary 2.1. By using theorem 2.2, 2.3, we obtain the fact that $\forall X \forall Y(\bar{N}(X \cup Y)=\bar{N}(X) \cup$ $\bar{N}(Y)) \Leftrightarrow$ There does not exist a pair $x, y[(|N(x)|>1) \wedge(|N(y)|>1) \wedge(x \in N(y)) \wedge(y \notin N(x))]$.

Proposition 2.3. Let $(U, C, N)$ be a Covering approximation space. The properties below hold.

$$
\begin{aligned}
& (1) \underline{N}(U-\underline{N}(X)) \subseteq U-\underline{N}(X) \\
& (2) \underline{N}(U-X) \subseteq U-\bar{N}(X)
\end{aligned}
$$

Generally speaking, equality $\underline{N}(U-\underline{N}(X))=U-\underline{N}(X)$ and $\underline{N}(U-X)=U-\bar{N}(X)$ do not always hold.

Example 2.3. Let $U=\{1,2,3,4\}, C=\{\{1\},\{2\},\{3\},\{3,4\}\}, X_{0}=\{1,2,3\}$. We have $\underline{N}(U-$ $\left.\underline{N}\left(X_{0}\right)\right)=\emptyset \neq U-\underline{N}\left(X_{0}\right)=\{4\}$

Example 2.4. Let $U=\{1,2,3,4,5\}, C=\{\{1\},\{2\},\{3\},\{1,2,3,4\},\{5\}\}, X_{0}=\{1,2,3\}$. We have $\underline{N}\left(U-X_{0}\right)=\{5\} \neq U-\bar{N}\left(X_{0}\right)=\{4,5\}$.

Theorem 2.4. Let $(U, C, N)$ be a Covering approximation space. $\forall X[\underline{N}(U-\underline{N}(X)) \subseteq(U-$ $\underline{N}(X))] \Leftrightarrow N$ forms a partition of $U$.

Proof. " $\Rightarrow$ ", proof by contradiction. Suppose $N$ does not form a partition of $U$. From proposition 2.1, we can obtain $x_{0}, y_{0} \in U$ such that $x_{0} \in N\left(y_{0}\right)$ and $y_{0} \notin N\left(x_{0}\right)$. If we choose $X_{0}=N\left(x_{0}\right)$, then $y_{0} \in U-\underline{N}\left(X_{0}\right)$ and $y_{0} \notin \underline{N}\left(U-\underline{N}\left(X_{0}\right)\right)$. This means $\underline{N}\left(U-\underline{N}\left(X_{0}\right)\right) \neq U-\underline{N}\left(X_{0}\right)$, contradicts the assumption of necessity.

$" \Leftarrow "$ is simple.

Theorem 2.5. Let $(U, C, N)$ be a Covering approximation space.

(1) $\forall X[\underline{N}(U-\underline{N}(X))=U-\underline{N}(X)] \Leftrightarrow N$ forms a partition of $U$.

$(2) \forall X[\underline{N}(U-\bar{X})=(U-\bar{N}(\bar{X}))] \Leftrightarrow N$ forms a partition of $U$.

Proof. (1)" $\Rightarrow "$, proof by contradiction. Suppose $N$ does not form a partition of $U$. From proposition 2.1, we can obtain $x_{0}, y_{0} \in U$ such that $x_{0} \in N\left(y_{0}\right)$ and $y_{0} \notin N\left(x_{0}\right)$. If we choose $X_{0}=N\left(x_{0}\right)$, then $y_{0} \in U-\underline{N}\left(X_{0}\right)$ and $y_{0} \notin \underline{N}\left(U-\underline{N}\left(X_{0}\right)\right)$. This means $\underline{N}\left(U-X_{0}\right) \neq \underline{N}\left(U-\underline{N}\left(X_{0}\right)\right)$, contradicts the assumption of necessity.

$" \Leftarrow "$ is simple.

$(2) " \Rightarrow "$, proof by contradiction. Suppose $N$ does not form a partition of $U$. From proposition 2.1, we can obtain $x_{0}, y_{0} \in U$ such that $x_{0} \in N\left(y_{0}\right)$ and $y_{0} \notin N\left(x_{0}\right)$. If we choose $X_{0}=N\left(x_{0}\right)$, 
then $y_{0} \in U-\bar{N}\left(X_{0}\right)$ and $y_{0} \notin \underline{N}\left(U-X_{0}\right)$. This means $\underline{N}\left(U-X_{0}\right) \neq\left(U-\bar{N}\left(X_{0}\right)\right)$, contradicts the assumption of necessity.

$" \Leftarrow "$ is simple.

\section{Relationships between the new type lower and upper approximation operations and the other types}

For a covering of $U$, there are about ten types of lower approximation operations and upper approximation operations. A natural question is that what are the relationships among them. To answer this question, we need to narrate the definitions of ten types of lower approximation operations and upper approximation operations.

Definition 3.1. Let $(U, C, N)$ be a Covering approximation space. For each $n \in\{1,2,3,4,5,6,7,8,9$, $10\}, \underline{C}_{n}$ and $\bar{C}_{n}$ are called the $n-t h$ lower approximation operation and upper approximation operation respectively, defined as follow:

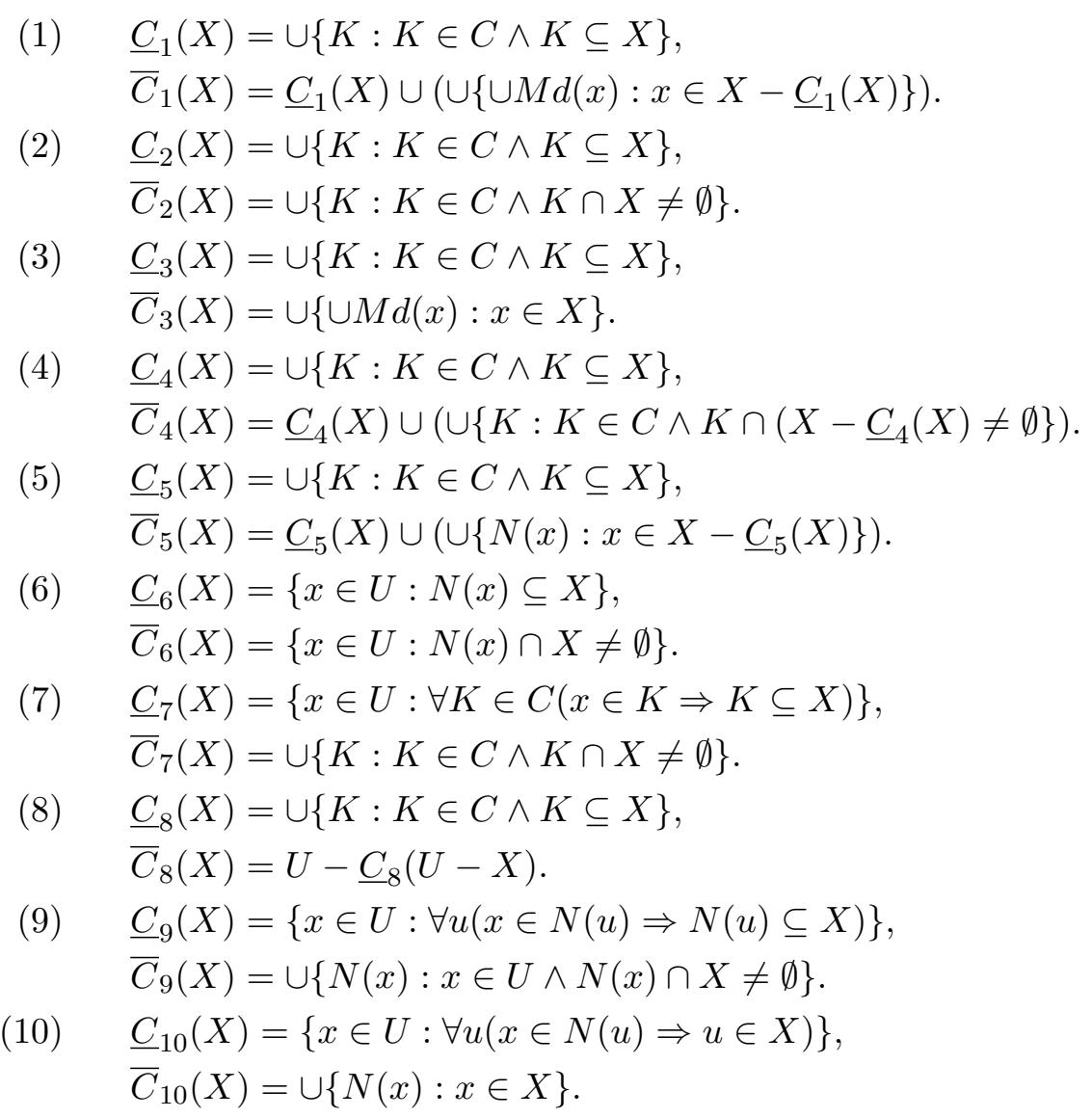

Remark 3.1. $\underline{C}_{n}$ and $\bar{C}_{n}(n=1,2,3)$ can be found from [19], $\underline{C}_{4}$ and $\bar{C}_{4}$ can be found from [21], $\underline{C}_{5}$ and $\bar{C}_{5}$ can be found from [18], $\underline{C}_{6}$ and $\bar{C}_{6}$ can be found from [21], $\underline{C}_{7}$ and $\bar{C}_{7}$ can be found from [25], $\underline{C}_{n}$ and $\bar{C}_{n}(n=8,9,10)$ can be found from [10].

Proposition 3.1. Let $(U, C, N)$ be a Covering approximation space. The properties below hold, 
but all the symbol" $\subseteq$ " can not be replaced by symbol"=".

(1) $\quad \forall X\left(\underline{C}_{1}(X) \subseteq \underline{N}(X)\right)$,

(2) $\left.\forall X(\bar{N}(X)) \subseteq \bar{C}_{2}(X)\right)$,

(3) $\left.\forall X(\bar{N}(X)) \subseteq \bar{C}_{4}(X)\right)$.

Example 3.1. Let $U=\{1,2,3,4,5\}, C=\{\{1,2\},\{3,4\},\{5\},\{3,5\}\}, X_{0}=\{1,2,3\}$. We have $\underline{N}\left(X_{0}\right)=\{1,2,3\} \neq \underline{C}_{1}\left(X_{0}\right)=\{1,2\}$.

Example 3.2. Let $U=\{1,2,3,4,5\}, C=\{\{1,2,3\},\{3,4,5\}\}, X_{0}=\{3\}$. We have $\bar{N}\left(X_{0}\right)=\{3\} \neq$ $\bar{C}_{2}\left(X_{0}\right)=\{1,2,3,4,5\}=\bar{C}_{4}\left(X_{0}\right)$.

Theorem 3.1. Let $(U, C, N)$ be a Covering approximation space.

$(1) \forall X\left(\underline{C}_{1}(X)=\underline{\underline{N}}(X)\right) \Leftrightarrow \forall x(|M d(x)|=1)$,

(2) $\forall X\left(\bar{C}_{1}(X)=\overline{\bar{N}}(X)\right) \Leftrightarrow[\forall x(|M d(x)|=1) \wedge N$ forms a partition of $U]$,

(3) $\forall X\left(\bar{C}_{2}(X)=\bar{N}(X)\right) \Leftrightarrow C$ forms a partition of $U$,

(4) $\forall X\left(\bar{C}_{3}(X)=\bar{N}(X)\right) \Leftrightarrow[\forall x(|M d(x)|=1) \wedge N$ forms a partition of $U]$,

$(5) \forall X\left(\bar{C}_{4}(X)=\bar{N}(X)\right) \Leftrightarrow\{[\forall x(|M d(x)|=1)] \wedge \forall K \in C[\forall y \in K(\{y\} \in C) \vee \forall z \in K(M d(z)=$ $\{K\})]\}$.

Proof. (1)" $\Rightarrow$ ", proof by contradiction. Suppose $\exists x_{0}\left(\left|M d\left(x_{0}\right)\right|>1\right)$, we can find $K_{1}, K_{2} \in M d\left(x_{0}\right)$ such that $x_{0} \in K_{1} \cap K_{2}, K_{1} \cap K_{2} \subsetneq K_{1}$ and $K_{1} \cap K_{2} \subsetneq K_{2}$. If we choose $X_{0}=K_{1} \cap K_{2}$, then $\underline{N}\left(X_{0}\right)=K_{1} \cap K_{2} \neq \underline{C}_{1}\left(X_{0}\right)=\emptyset$. This contradicts the assumption of necessity.

$" \Leftarrow "$ is simple.

$(2) " \Rightarrow$ ", proof by contradiction. Firstly, we prove $\forall x(|M d(x)|=1)$. Suppose $\exists x_{0}\left(\left|M d\left(x_{0}\right)\right|>\right.$ 1 ), select $K_{1}, K_{2} \in M d\left(x_{0}\right)$ such that $x_{0} \in K_{1} \cap K_{2}, K_{1} \cap K_{2} \subsetneq K_{1}$ and $K_{1} \cap K_{2} \subsetneq K_{2}$. Without loss of generality, if we choose $y_{0} \in K_{2}$ and $y_{0} \notin K_{1}$, then $y_{0} \notin \bar{N}\left(K_{1} \cap K_{2}\right)$. Since there does not exist $K \in C$ such that $x_{0} \in K \subseteq K_{1} \cap K_{2}$, so $x_{0} \in K_{1} \cap K_{2}-\underline{C}_{1}\left(K_{1} \cap K_{2}\right)$ and $y_{0} \in \bar{C}_{1}\left(K_{1} \cap K_{2}\right)$. This means $\bar{N}\left(K_{1} \cap K_{2}\right) \neq \bar{C}_{1}\left(K_{1} \cap K_{2}\right)$, contradicts the assumption of necessity.

Secondly, we prove that $N$ forms a partition of $U$. Suppose $N$ is not a partition of $U$, by proposition 2.1, $\exists x_{1}, y_{1} \in U$ such that $x_{1} \in N\left(y_{1}\right)$ and $y_{1} \notin N\left(x_{1}\right)$. If we choose $X_{0}=N\left(y_{1}\right)-$ $N\left(x_{1}\right)$, then $x_{1} \notin N\left(X_{0}\right)$. Since $x_{1} \in N\left(y_{1}\right)$, so there dose not exist $K \in C$ such that $y_{1} \in K \subseteq X_{0}$. Thus $y_{1} \in X_{0}-\underline{C}_{1}\left(X_{0}\right)$ and $x_{1} \in \cup M d\left(y_{1}\right) \subseteq \bar{C}_{1}\left(X_{0}\right)$. This means $\bar{C}_{1}\left(X_{0}\right) \neq \bar{N}\left(X_{0}\right)$, contradicts the assumption of necessity.

$" \Leftarrow ", \forall X \subseteq U$, by theorem 3.1(1) and $\forall x(|M d(x)|=1)$, we have $\underline{C}_{1}(X)=\underline{N}(X)$ and $\forall y(N(y)=$ $\cup M d(y)) . \forall z \in \bar{C}_{1}(X)$, we take two conditions into consideration: $(\sharp) z \in \underline{C}_{1}(X) \subseteq X$, we have $z \in \bar{N}(X)$. (घ) $\exists z_{0} \in X-\underline{C}_{1}(X)$ such that $z \in \cup M d\left(z_{0}\right)=N\left(z_{0}\right)$. Since $N$ is a partition of $U$, so $N(z)=N\left(z_{0}\right)$. This means $z_{0} \in N(z) \cap\left(X-\underline{C}_{1}(X)\right)=N(z) \cap(X-\underline{N}(X) \neq \emptyset$. By the definition of $\bar{N}(X)$, We have $z \in \bar{N}(X)$. Coming $(\sharp)$ with $(\natural), \bar{C}_{1}(X) \subseteq \bar{N}(X)$. On the other hand,

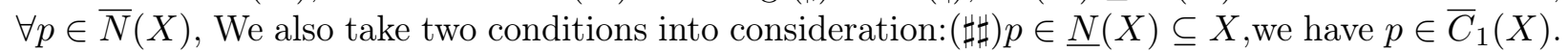
(吅) $N(p) \cap(X-\underline{N}(X)) \neq \emptyset$, We can choose $p_{0} \in N(p) \cap(X-\underline{N}(X))=N(p) \cap\left(X-\underline{C}_{1}(X)\right)$, consider that $N$ is a partition of $U$, thus $p \in N\left(p_{0}\right)=\cup M d\left(p_{0}\right)$. By the definition of $\bar{C}_{1}(X)$, we have $p \in \bar{C}_{1}(X)$. Coming $(\sharp)(\sharp)$ with $(\sharp \sharp)($ (甘) $), \bar{N}(X)=\bar{C}_{1}(X)$ holds.

$(3)$, the proof of (3) is simple.

(4), the proof of (4) is similar to (2) .

(5), " $\Rightarrow$ ", proof by contradiction. Firstly, we prove $\forall x(|M d(x)|=1)$. Suppose $\exists x_{0}\left(\left|M d\left(x_{0}\right)\right|>\right.$ 1 ), select $K_{1}, K_{2} \in M d\left(x_{0}\right)$ such that $x_{0} \in K_{1} \cap K_{2}, K_{1} \cap K_{2} \subsetneq K_{1}$ and $K_{1} \cap K_{2} \subsetneq K_{2}$. Without 
loss of generality, if we choose $y_{0} \in K_{2}$ and $y_{0} \notin K_{1}$, then $y_{0} \notin \bar{N}\left(K_{1} \cap K_{2}\right)$. Since there does not exist $K \in C$ such that $x_{0} \in K \subseteq K_{1} \cap K_{2}$, so $x_{0} \in K_{1} \cap K_{2}-\underline{C}_{4}\left(K_{1} \cap K_{2}\right)$ and $y_{0} \in \bar{C}_{4}\left(K_{1} \cap K_{2}\right)$. This means $\bar{N}\left(K_{1} \cap K_{2}\right) \neq \bar{C}_{4}\left(K_{1} \cap K_{2}\right)$, contradicts the assumption of necessity.

Secondly, we prove $\forall K \in C[\forall y \in K(\{y\} \in C) \vee \forall z \in K(M d(z)=\{K\})] . \forall K \in C$, we take two conditions into consideration: $(\sharp) \exists p_{0} \in K\left(\left\{p_{0}\right\} \in C\right) \Rightarrow \forall y \in K(\{y\} \in C)$. Otherwise, $\exists q_{0} \in K\left(M d\left(q_{0}\right)=\left\{K_{3}\right\} \wedge\left|K_{3}\right|>1\right)$. If select $q^{\prime} \in K_{3}, q^{\prime} \neq q_{0}$ and let $Y_{0}=K_{3}-\left\{q^{\prime}, p_{0}\right\}$, then $p_{0} \notin \bar{N}\left(Y_{0}\right)$ and $p_{0} \in \bar{C}_{4}\left(Y_{0}\right)$. This means $\bar{N}\left(Y_{0}\right) \neq \bar{C}_{4}\left(Y_{0}\right)$, contradicts the assumption of necessity. (घ) $\forall m \in K(\{m\} \notin C) \Rightarrow \forall n \in K(M d(n)=\{K\})$. Otherwise, $\exists m_{0} \in K$ such that $M d\left(m_{0}\right)=\left\{K_{4}\right\},\left|K_{4}\right|>1$ and $K_{4} \varsubsetneqq K$. Select $n_{0} \in K-K_{4}, m^{\prime} \in K_{4}, m^{\prime} \neq m_{0}$ and let $Z_{0}=\left\{n_{0}\right\}$, we obtain that $m_{0} \notin \bar{N}\left(Z_{0}\right)$ and $m_{0} \in \bar{C}_{4}\left(Z_{0}\right)$. This means $\bar{C}_{4}\left(Z_{0}\right) \neq \bar{N}\left(Z_{0}\right)$, contradicts the assumption of necessity.

$" \Leftarrow "$ is simple.

Proposition 3.2. Let $(U, C, N)$ be a Covering approximation space. The properties below hold, but all the symbol" $\subseteq$ " can not be replaced by symbol" $="$.

(1) $\forall X\left(\underline{C}_{7}(X) \subseteq \underline{N}(X)\right)$,

(2) $\left.\forall X(\bar{N}(X)) \subseteq \bar{C}_{6}(X)\right)$.

Example 3.3. Let $U=\{1,2,3,4\}, C=\{\{1,2,3\},\{1,2,4\}\}, X_{0}=\{1,2\}$. We have $\underline{N}\left(X_{0}\right)=$ $\{1,2\} \neq \underline{C}_{7}\left(X_{0}\right)=\emptyset$.

Example 3.4. Let $U=\{1,2,3,4\}, C=\{\{1\},\{2\},\{3,4\},\{4,5\}\}, X_{0}=\{4\}$. We have $\bar{N}\left(X_{0}\right)=$ $\{4\} \neq \bar{C}_{6}\left(X_{0}\right)=\{3,4,5\}$.

Theorem 3.2. Let $(U, C, N)$ be a Covering approximation space.

$(1) \forall X\left(\underline{C}_{7}(X)=\underline{N}(X)\right) \Leftrightarrow C$ forms a partition of $U$,

(2) $\forall X\left(\bar{C}_{5}(X)=\overline{\bar{N}}(X)\right) \Leftrightarrow N$ forms a partition of $U$,

(3) $\forall X\left(\bar{C}_{6}(X)=\bar{N}(X)\right) \Leftrightarrow N$ forms a partition of $U$.

Proof. (1) the proof of (1) is simple.

$(2) " \Rightarrow "$, proof by contradiction. Suppose $N$ is not a partition of $U$, by proposition 2.1, $\exists x_{0} \exists y_{0}\left(x_{0} \in N\left(y_{0}\right) \wedge y_{0} \notin N\left(x_{0}\right)\right)$. If we let $X_{0}=N\left(y_{0}\right)-N\left(x_{0}\right)$, then $x_{0} \notin \bar{N}\left(X_{0}\right)$ and $x_{0} \in \bar{C}_{5}\left(X_{0}\right)$. This means $\bar{C}_{5}\left(X_{0}\right) \neq \bar{N}\left(X_{0}\right)$, contradicts the assumption of necessity.

"६", $\forall X \subseteq U$. Firstly, we prove $\bar{N}(X) \subseteq \bar{C}_{5}(X) . \forall x \in \bar{N}(X)$, we take two conditions into consideration: $(\sharp), x \in \underline{N}(X)$, we have $x \in X \subseteq \bar{C}_{5}(X)$. (দ) $N(x) \cap(X-\underline{N}(X)) \neq \emptyset$, take $x_{0} \in N(x) \cap(X-\underline{N}(X))$, from proposition $3.1(1), x_{0} \in X-\underline{N}(X) \subseteq X-\underline{C_{5}}(X)$ holds. By the assumption that $N$ is a partition of $U$, we have $N\left(x_{0}\right)=N(x)$. According to the definition of $\bar{C}_{5}(X)$, we have $x \in \bar{C}_{5}(X)$, this means $\bar{N}(X) \subseteq \bar{C}_{5}(X)$. Secondly, we prove $\bar{C}_{5}(X) \subseteq \bar{N}(X)$. $\forall y \in \bar{C}_{5}(X)$, we also take two conditions into consideration: ( $\left.\sharp \sharp\right) y \in X$, we have $y \in \bar{N}(X)$. (如) $y \in \bar{C}_{5}(X)-X, \exists y_{0} \in X-\underline{C}_{5}(X)$ such that $y \in N\left(y_{0}\right)$. By the assumption that $N$ is a partition of $U$, we have $N(y)=N\left(y_{0}\right)$. That is to say $y_{0} \in X-\underline{N}(X)$ and $y_{0} \in N(y) \cap(X-\underline{N}(X)) \neq \emptyset$. By the definition of $\bar{N}(X)$, we have $y \in \bar{N}(X)$. This means $\bar{C}_{5}(X) \subseteq \bar{N}(X)$. Therefore $\bar{C}_{5}(X)=\bar{N}(X)$ holds.

$(3) " \Rightarrow "$, proof by contradiction. Suppose $N$ is not a partition of $U$, by proposition 2.1, $\exists x_{0} \exists y_{0}\left(x_{0} \in N\left(y_{0}\right) \wedge y_{0} \notin N\left(x_{0}\right)\right)$. If we let $X_{0}=N\left(x_{0}\right)$, then $y_{0} \in \bar{C}_{6}\left(X_{0}\right)$ and $y_{0} \notin \bar{N}\left(X_{0}\right)$. this means $\bar{C}_{6}\left(X_{0}\right) \neq \bar{N}\left(X_{0}\right)$, contradicts the assumption of necessity. 
$" \Leftarrow "$ is simple.

Proposition 3.3. Let $(U, C, N)$ be a Covering approximation space. The properties below hold, but all the symbol" $\subseteq$ " can not be replaced by symbol" $="$.

(1) $\forall X\left(\underline{C}_{9}(X) \subseteq \underline{N}(X)\right)$

(2) $\left.\forall X(\bar{N}(X)) \subseteq \bar{C}_{8}(X)\right)$,

(3) $\left.\forall X(\bar{N}(X)) \subseteq \bar{C}_{9}(X)\right)$.

Example 3.5. Let $U=\{1,2,3,4,5\}, C=\{\{1,2,3\},\{1,2,4\},\{1,2,3,4,5\}\}, X_{0}=\{1,2,3\}$. We have $\underline{N}\left(X_{0}\right)=\{1,2,3\} \neq \underline{C}_{9}\left(X_{0}\right)=\emptyset$.

Example 3.6. Let $U=\{1,2,3,4,5\}, C=\{\{1,2\},\{1,2,3,4,5\}\}, X_{0}=\{1,2\}$. We have $\bar{N}\left(X_{0}\right)=$ $\{1,2\} \neq \bar{C}_{8}\left(X_{0}\right)=\{1,2,3,4,5\}$.

Example 3.7. Let $U=\{1,2,3,4\}, C=\{\{1\},\{2\},\{3,4\},\{4\}\}, X_{0}=\{3\}$. We have $\bar{N}\left(X_{0}\right)=\{3\} \neq$ $\bar{C}_{9}\left(X_{0}\right)=\{3,4\}$.

Theorem 3.3. Let $(U, C, N)$ be a Covering approximation space.

(1) $\forall X\left(\underline{C}_{9}(X)=\underline{N}(X)\right) \Leftrightarrow N$ forms a partition of $U$,

(2) $\forall X\left(\bar{C}_{8}(X)=\overline{\bar{N}}(X)\right) \Leftrightarrow[\forall x(|M d(x)|=1) \wedge N$ forms a partition of $U]$,

(3) $\forall X\left(\bar{C}_{9}(X)=\bar{N}(X)\right) \Leftrightarrow N$ forms a partition of $U$,

(4) $\forall X\left(\bar{C}_{10}(X)=\bar{N}(X)\right) \Leftrightarrow N$ forms a partition of $U$.

Proof. (1), " $\Rightarrow$ ", proof by contradiction. Suppose $N$ is not a partition of $U$, by proposition 2.1, $\exists x_{0} \exists y_{0}\left(x_{0} \in N\left(y_{0}\right) \wedge y_{0} \notin N\left(x_{0}\right)\right)$. If we let $X_{0}=N\left(x_{0}\right)$, then $x_{0} \in \underline{N}\left(X_{0}\right)$ and $x_{0} \notin \underline{C}_{9}\left(X_{0}\right)$. This means $\underline{N}\left(X_{0}\right) \neq \underline{C}_{9}\left(X_{0}\right)$, contradicts the assumption of necessity.

$" \Leftarrow "$ is simple.

(2), " $\Rightarrow$ ", proof by contradiction. Firstly, we prove $\forall x(|M d(x)|=1)$. Suppose $\exists x_{0}\left(\left|M d\left(x_{0}\right)\right|>\right.$ $1)$, we can find out $K_{1}, K_{2} \in M d\left(x_{0}\right)$ such that $x_{0} \in K_{1} \cap K_{2}, K_{1} \cap K_{2} \varsubsetneqq K_{1}$ and $K_{1} \cap K_{2} \varsubsetneqq$ $K_{2}$. By the assumption that $\forall X\left(\bar{C}_{8}(X)=\bar{N}(X)\right)$ and the fact that $\bar{N}\left(K_{1} \cap K_{2}\right)=K_{1} \cap K_{2}$, $\exists L_{1}, L_{2}, \cdots L_{n} \in C$ such that $U-\left(K_{1} \cap K_{2}\right)=L_{1} \cup L_{2} \cup \cdots \cup L_{n}$. Since $\bar{N}\left(L_{1} \cup L_{2} \cup \cdots \cup L_{n}\right)=$ $L_{1} \cup L_{2} \cup \cdots \cup L_{n}=U-\left(K_{1} \cap K_{2}\right)$, so $\exists L^{1}, L^{2}, \cdots L^{m} \in C$ such that $K_{1} \cap K_{2}=L^{1} \cup L^{2} \cup \cdots \cup L^{m}$. This means $\exists i_{0} \in\{1,2, \cdots, m\}$ such that $x_{0} \in L^{i_{0}} \subseteq K_{1} \cap K_{2} \varsubsetneqq K_{2}$, contradicts the fact that $K_{2} \in M d\left(x_{0}\right)$. Secondly, we prove that $N$ is a partition of $U$. Otherwise, by proposition 2.1, $\exists y_{0} \exists z_{0}\left(y_{0} \in N\left(z_{0}\right) \wedge z_{0} \notin N\left(y_{0}\right)\right)$. If we let $X_{0}=N\left(y_{0}\right)$, then $z_{0} \in \bar{C}_{8}\left(X_{0}\right)$ and $z_{0} \notin \bar{N}\left(X_{0}\right)$. This means $\bar{C}_{8}\left(X_{0}\right)=\bar{N}\left(X_{0}\right)$, contradicts the assumption of necessity.

$" \Leftarrow " . \forall X \subseteq U$, by proposition 3.3(2), we only need to prove $\bar{C}_{8}(X) \subseteq \bar{N}(X) . \forall x \in \bar{C}_{8}(X)$, we take two conditions into consideration: $(\sharp) x \in X$, we have $x \in \bar{N}(X)$. ( $(\natural) x \in \bar{C}_{8}(X)-X$, since $\forall y(|M d(y)|=1)$, so $\cup M d(x)=N(x)$ and $N(x) \cap X \neq \emptyset$. We can select $x_{0} \in N(x) \cap X$, by the condition that $N$ is a partition of $U$, we have $N(x)=N\left(x_{0}\right)$ and $N(x)=N\left(x_{0}\right) \nsubseteq X$. This means $x_{0} \notin \underline{N}(X)$ and $x_{0} \in N(x) \cap(X-\underline{N}(X)) \neq \emptyset$. From the definition of $\bar{N}(X)$, we have $x \in \bar{N}(X)$. According to $(\sharp)(\natural)$, we finally have $\bar{C}_{8}(X) \subseteq \bar{N}(X)$.

(3), " $\Rightarrow$ ", proof by contradiction. Suppose $N$ is not a partition of $U$, by proposition 2.1, $\exists x_{0} \exists y_{0}\left(x_{0} \in N\left(y_{0}\right) \wedge y_{0} \notin N\left(x_{0}\right)\right)$. If we let $X_{0}=N\left(x_{0}\right)$, then $y_{0} \in \bar{C}_{9}\left(X_{0}\right)$ and $y_{0} \notin \bar{N}\left(X_{0}\right)$. This means $\bar{C}_{9}\left(X_{0}\right) \neq \bar{N}\left(X_{0}\right)$, contradicts the assumption of necessity.

$" \Leftarrow "$ is simple. 
(4), " $\Rightarrow$ ", proof by contradiction. Suppose $N$ is not a partition of $U$, by proposition 2.1, $\exists x_{0} \exists y_{0}\left(x_{0} \in N\left(y_{0}\right) \wedge y_{0} \notin N\left(x_{0}\right)\right)$. If we let $X_{0}=N\left(y_{0}\right)-N\left(x_{0}\right)$, then $x_{0} \in \bar{C}_{10}\left(X_{0}\right)$ and $x_{0} \notin \bar{N}\left(X_{0}\right)$. This means $\bar{C}_{10}\left(X_{0}\right) \neq \bar{N}\left(X_{0}\right)$, contradicts the assumption of necessity.

$" \Leftarrow "$ is simple.

In order to show the structures of $\underline{N}(X)$ and $\bar{N}(X)$ more clearly, we introduce the conception of Alexander topological space. Let $(U, C, N)$ be a covering approximation space. As a topological base, $N$ can induce a topology $T$ on $U$. the topological space $(U, T)$ is called Alexander topological space.

$\forall X \subseteq U$, let symbol $\operatorname{int}(X)$ represent the interior of $X$ and $\operatorname{cl}(X)$ represent the closure of $X$, then

$$
\begin{aligned}
& \underline{N}(X)=\operatorname{int}(X), \\
& \bar{N}(X)=\operatorname{int}(X) \cup \operatorname{cl}(X-\operatorname{int}(X))
\end{aligned}
$$

As the end, we introduce definitions of $n-t h$ inner and outer accuracy to show the reason why we introduce the type of covering-based generalized rough set.

Definition 3.2. Let $(U, C, N)$ be a Covering approximation space. For a subset $X$ of $U$,denote $\rho_{i}(X)=\frac{\left|C_{i}(X)\right|}{|X|}(i \in\{1,2, \cdots, 10\})$ the $n-t h$ inner accuracy of $X$, and $\rho^{i}(X)=\frac{\left|\bar{C}^{i}(X)\right|}{|X|}(i \in$ $\{1,2, \cdots, 10\})$ the $n-t h$ outer accuracy of $X$, where symbol $|$.$| represents the cardinality of a$ set. For $i=0$, denote $\rho_{0}(X)=\frac{\left.\mid \underline{N}_{(} X\right) \mid}{|X|}$ and $\rho^{0}(X)=\frac{\left.\mid \bar{N}_{(} X\right) \mid}{|X|}$.

From the definition 3.2, we easily see that $\rho_{i}(X) \leqslant 1$ for each $i$ and $X$, and $\rho^{i}(X) \geqslant 1$ for each $i$ and $X$. For a fixed subset $X$ of $U$, if $\rho_{i}(X) \geqslant \rho_{j}(X)$,we say the $i-t h$ inner accuracy of $X$ is higher than the $j$-th inner accuracy of $X$, similarly, if $\rho^{i}(X) \leqslant \rho^{j}(X)$, we say the $i$-th outer accuracy of $X$ is higher than the $j-t h$ outer accuracy of $X$.

Theorem 3.4. Let $(U, C, N)$ be a Covering approximation space.

(3) $\quad \forall X\left(\rho_{0}(X) \geqslant \rho_{9}(X)\right)$

(4) $\forall X\left(\rho^{0}(X) \leqslant \rho^{2}(X)\right)$,

(5) $\forall X\left(\rho^{0}(X) \leqslant \rho^{4}(X)\right)$,

(6) $\forall X\left(\rho^{0}(X) \leqslant \rho^{6}(X)\right)$,

(7) $\forall X\left(\rho^{0}(X) \leqslant \rho^{8}(X)\right)$,

(8) $\quad \forall X\left(\rho^{0}(X) \leqslant \rho^{9}(X)\right)$.

Proof. Straightforwardly by proposition 3.1, proposition 3.2, and proposition 3.3.

Definition 3.2 and Theorem 3.4 indicate that the type of covering-based rough set possesses a well inner and outer accuracy, this is the meaning we propose this kind of covering-based rough set. 


\section{Conclusions}

In this paper, we have presented a new type of covering-based generalized rough set, and proved some properties of $\underline{N}(X)$ and $\bar{N}(X)$. Here, we could not obtain the sufficient and necessary condition for $\forall X(\bar{N}(U-\bar{N}(X))=U-\bar{N}(X))$. We mainly discussed the sufficient and necessary conditions for $\forall X\left(\underline{C}_{i}(X)=\underline{N}(X)\right)$ and $\forall X\left(\bar{C}_{i}(X)=\bar{N}(X)\right)(i=\{1,2, \cdots, 10\})$. The most important sufficient and necessary condition is that $N$ forms a partition of $U$. This article introduces two interesting questions: (1) What conditions of $C$ should be satisfied can infer that $N$ is a partition of $U$ ? (2) What conditions $\underline{N}(X)$ or $\bar{N}(X)$ should be satisfied can infer that $N$ is a partition of $U$ ? Solving problem (1) and (2) will be our future work.

\section{Acknowledgment}

This work is supported by (No.G2018004)

\section{Reference}

[1] G. Cattaneo, Abstract approximation spaces for rough theory, in: Rough Sets in Knowledge Discovery 1: Methodology and Applications, 1998, pp. 59-98.

[2] G. Cattaneo, D. Ciucci, Algebraic structures for rough sets, in: LNCS, vol. 3135, 2004, pp. 208-252.

[3] M. Kondo, On the structure of generalized rough sets, Information Sciences 176 (5) (2005) 589-600.

[4] K. Qin, Z. Pei, On the topological properties of fuzzy rough sets, Fuzzy Sets and Systems 151 (3) (2005) 601-613.

[5] A. Skowron, J. Stepaniuk, Tolerance approximation spaces, Fundamenta Informaticae 27 (1996) 245-253.

[6] R. Slowinski, D. Vanderpooten, A generalized definition of rough approximations based on similarity, IEEE Transactions on Knowledge and DataEngineering 12 (2) (2000) 331-336

[7] Y. Yao, A comparative study of fuzzy sets and rough sets, Information Sciences 109 (1998) 227-242.

[8] Y. Yao, Constructive and algebraic methods of theory of rough sets, Information Sciences 109 (1998) 21-47.

[9] L.A. Zadeh, Fuzzy sets, Information and Control 8 (1965) 338-353.

[10] K. Qin, Y. Gao and Z. Pei, On covering rough sets, LNAI,4481(2007), 34-41.

[11] S.Comer, An algebraic approach to the approximation of information, Fundamenta Informaticae 14 (1991) 492-502.

[12] Z. Pawlak, Rough Sets: Theoretical Aspects of Reasoning about Data, Kluwer Academic Publishers, Boston, 1991.

[13] Z. Bonikowski, Algebraic structure of Rough sets, Rough sets fuzzy sets and knowledge discovery, SpringerVerlag, london,1994,pp.242-247

[14] Z. Pawlak, A. Skowron, Rough sets: some extensions, Information Sciences 177 (1) (2007) 28-40.

[15] X.Chen, Q.Li, Construction of rough approximations in fuzzy setting, Fuzzy sets and systems 158 (2007) 26412653.

[16] L. Zadeh, Fuzzy logic = computing with words, IEEE Transactions on Fuzzy Systems 4 (1996) 103-111.

[17] W. Zhu, F.-Y. Wang, Properties of the third type of covering-based rough sets, in: ICMLC07, Hong Kong, China, 19-22 August, 2007, pp. 3746-3751.

[18] W. Zhu, Topological approaches to covering rough sets, Information Sciences 177 (6) (2007) 1499-1508.

[19] W. Zhu, F.-Y. Wang, On three types of covering rough sets, IEEE Transactions on Knowledge and Data Engineering 19 (8) (2007) 1131-1144.

[20] G.Liu, Axiomatic systems for rough set over fuzzy rough sets, International journal of approximate reasoning 48 (2008) 857-867.

[21] Catteneo,G, Generalized rough sets, Studia Logic 58 (1997) 47-77.

[22] W. Zhu, Relationship among basic concepts in covering-based rough sets, Information Sciences 179 (14) (2009) 2478-2486.

[23] W. Zhu, F.-Y. Wang, The fourth type of covering-based rough sets, Information Sciences 1016(10)(2012)1-13.

[24] Nianbai Fan, Gongzhu Hu, Hui Liu. Study of Definable Subsets in Covering Approximation Space of Rough Sets. Proceedings of the 2011IEEE International Conference on Information Reuse and Integration 1(5) (2011) $21-24$. 
[25] Xun Ge and Zhaowen Li. Definable subset in covering approximation spaces. International Journal of Computational and Mathematical Sciences, 5(1)(2011) 31-34.

[26] Nianbai Fan, Gongzhu Hu, Wei Zhang. Study on conditions of neighborhoods forming a partition, Fuzzy Systems and Knowledge Discovery (FSKD), 2012 9th International Conference 256-259.

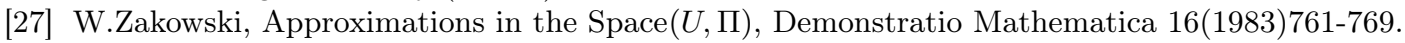

[28] D.G.Chen, C.Z. Wang A new approach to arrtibute reduction of consistent and inconsistent covering decision systems with covering rough sets, Inf.Sci.176(2007)3500-3518

[29] X.Ge An application of covering approximation spaces on network security, Comput.Math.Appl,60(2010)11911199.

[30] X.Ge Connectivity of covering approximation spaces and its applications onepidemiological issue, Appl.Soft comput,25(2014)445-451.

[31] T. Yang, Q. Li,B. Zhou Reduction about approximation spaces of covering generalized rough sets. Int. J. Approx. Reason, 51(2010):335šC345.

[32] J. Zhan, W. Xu Two types of covering based nultigranulation rough fuzzy sets and applications to decision making, Artificial Intelligence Review,2018, https://doi.org/10.1007/s10462-018-9649-8.

[33] J. Zhan, Q. Wang, Certain types of soft coverings based rough sets with applications, Int. J. Mach. Learn. Cybern.,2018,https://doi.org/10.1007/s13042-018-0785-x.

[34] L. Zhang, J. Zhan, J.C.R. Alcantud, Novel classes of fuzzy soft $\beta$-coverings-based fuzzy rough sets with applications to multi-criteria fuzzy group decision making, soft computing,2018, https://doi.org/10.1007/s00500018-3470-9.

[35] J. Zhan, J.C.R. Alcantud, A novel type of soft rough covering and its application to multicriteria group decision making, Artificial Intelligence Review,2018, https://doi.org/10.1007/s10462-018-9617-3.

[36] D'eer, Lynn, et. al., Neighborhood operators for covering-based rogh sets, Informaiton Sciences, 336(2016):2144.

[37] D'eer, Lynn, Chris Cornelis, and Y.Y. Yao, A semantically sound approach to Pawlak rough sets and coveringbased rough sets, Int. J. Approx. Reason.,78(2016):62-72.

[38] José Carlos R. Alcantud, Some formal relationships among soft sets, fuzzy sets, and their extensions, Int. J. Approx. Reason.,68(2016):45-53.

[39] José Carlos R. Alcantud, Fuzzy techniques for Decision Making, Symmetry,10(1):6(2018).

[40] Przemyslaw Grzegorzewski, On separability of Fuzzy relations, International Journal of Fuzzy Logic and Intelligent systems, $17(3): 6(2017): 137-144$. 